\title{
基于集成学习的风云四号遥感图像云相态分类算法
}

\author{
高 军 ${ }^{1,2}$, 陈 建 $^{1}$, 田晓宇 ${ }^{1}$
}

(1. 上海海事大学 信息工程学院, 上海 201306; 2. 西藏自治区经济和信息化厅信息化推进处, 拉萨 850033)

\begin{abstract}
摘要: 云相态分类在气象预报和气候研究中具有重要的地位。我国新一代气象卫星风云四号的成像仪 在光谱通道数量和空间分辨率较上一代风云二号有较大提升, 这为云相态的研究提供了新的遥感数 据。本文首先对风云四号相隔 $15 \mathrm{~min}$ 的遥感图像进行分析, 然后提出亮温云相态指数, 该指数可以进 行初步云相态分类, 最后在此基础上提出基于集成学习的云相态分类算法。实验结果与风云四号官方 云相态分类结果进行比较，水云的一致率达到 $91.69 \%$, 冰云的一致率达到 $76.10 \%$ 。
\end{abstract}

关键词: 云相态; 集成学习; 风云四号; 遥感图像处理

中图分类号：TP389.1 文献标识码：A 文章编号：1001-8891(2020)01-0068-07

\section{Ensemble-learning-based Cloud Phase Classification Method for FengYun-4 Remote Sensing Images}

\author{
GAO Jun ${ }^{1,2}$, CHEN Jian ${ }^{1}$, TIAN Xiaoyu ${ }^{1}$ \\ (1.College of Information Engineering, Shanghai Maritime University, Shanghai 201306, China;
}

2. Information Promotion Office, Department of Economy and Information Technology of Tibet Autonomous Region, Lasa 850033, China)

\begin{abstract}
Cloud phase classification plays an important role in meteorological forecast and climate research. The image of meteorological satellite FengYun-4 (FY-4) has more channels and better resolution than FY-2. So it provides new remote sensing data for the study of the cloud phase. This study uses a brightness temperature cloud phase index to obtain cloud phase data. Thereafter, using the cloud phase data and ensemble learning algorithm, we develop a cloud phase classification model. By applying the cloud phase classification model, the predicted classification accuracy of water cloud and ice cloud are $91.69 \%$ and $76.10 \%$, respectively.
\end{abstract}

Key words: cloud phase, ensemble learning, FY-4, remote image processing

\section{0 引言}

云相态是指云所处的热力学状态, 分为液态或固 态。云相态变化对地球大气辐射系统收支平衡、天气 系统的形成与演变都有不可忽视的影响, 因此研究云 相态对探究全球大气变化机理, 预测飞机积冰和反演 云微物理性质等都具有现实意义 ${ }^{[1-3]}$ 。

卫星遥感技术具有观测范围广、不受时空限制的 优点, 越来越多的人利用卫星遥感数据进行云相态分 类。根据卫星成像仪观测数据特性的不同, 国内外研 究者提出了许多云相态分类算法。主要的云相态分类 方法分为 2 种, 一种为光谱阈值法, 另一种为机器学 习算法。光谱阈值法一直被广泛使用, 包括经典的热 红外波段三光谱法 ${ }^{[4]}$, 以及后来经过三光谱法改进的
双光谱法 ${ }^{[3]}$, 还有仅利用热红外单通道的单光谱阈值 法 ${ }^{[5]}$ 。除了使用热红外波段, 可见光和近红外波段也 被用来进行云相态分类, 包括光谱比值 ${ }^{[6]}$ 和光谱对应 的云微物理特性 ${ }^{[7]}$ 等方法。单独使用热红外波段或者 可见光和近红外波段有一定限制, 研究者渐渐联合使 用可见光, 近红外和热红外波段 ${ }^{[8]}$ 来提高云相态分类 的准确性。刘建 ${ }^{[9-10]}$ 等人结合云微物理特性在风云一 号和风云二号上使用多光谱阈值法进行云相态分类。 Himawari 气象卫星官方 ${ }^{[11]}$ 也使用多光谱阈值法进行 云相态分类。光谱阈值法中, 无论是三光谱法, 双光 谱法, 多光谱法等, 阈值选取上都有一定的主观性且 阈值的大小与具体的遥感数据有关, 易受经纬度, 季 节, 气候等影响。

随着机器学习的快速发展, 许多研究者使用机器 
学习相关算法进行云相态分类 ${ }^{[12]}$, 神经网络算法由于 非线性拟合性突出被大量使用。从参数优化的反向传 播神经网络 ${ }^{[13]}$ 、经过特征篮选的 BP 神经网络 (Back

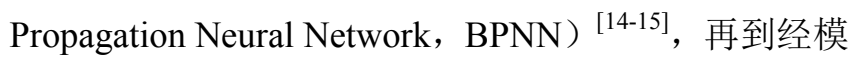
拟退火算法改进的 BP 神经网络 ${ }^{[16]}$, 还有自组织映射 网络 ${ }^{[17]}$ 。神经网络的使用降低了建立云相态分类模型 所需的成本, 减少了云相态分类所需的时间。但是 BP 神经网络属于有监督学习范畴, 训练该神经网络需要 大量有标签的数据, 大部分研究者对 BP 神经网络训 练时都使用了官方已经标注的云相态数据。自组织映 射网络属于无监督学习, 训练时不需要使用标签数据, 但是训练数据量很大时, 自组织映射网络神经元数量 也随之增加, 计算复杂度较高。

风云四号 $\mathrm{A}$ 星（FY-4A）属于新一代静止气象卫 星, 其搭载的多通道扫描成像辐射计 (Advanced Geosynchronous Radiation Imager, AGRI）为云相态分 类提供了新的数据来源。相比风云二号辐射成像仪, AGRI 的观测性能有显著提高, 其中, 辐射成像仪观 测通道从 5 个扩展到 14 个 (6 个可见/近红外波段、2 个中波红外波段、2 个水汽波段和 4 个长红外波段）, 观测时效从半小时提高到 $15 \mathrm{~min}$, 最高空间分辨率从 $1.25 \mathrm{~km}$ 提高到 $500 \mathrm{~m}$ 。AGRI 的观测通道数量与国际 同类卫星相比水平相当 ${ }^{[18-22]}$ 。

本文针对风云四号成像仪的多通道和相对于观测 区域静止的特性提出一种基于集成学习的云相态分类 方法, 此方法包括两步:

1) 根据风云四号特性提出亮温云相态指数, 并应 用该方法建立云相态数据集。

2) 使用基于集成学习的云相态分类算法, 应用云 相态数据集进行训练, 实现云相态分类。

\section{1 数据集建立}

\section{1 数据来源}

本文实验数据主要源于风云四号 AGRI 成像仪的 数据。表 1 列出了风云四号 AGRI 成像仪的通道设置。

风云四号 AGRI 的数据可以从国家卫星气象中心 数据服务网获取。截止到目前, 风云四号 $\mathrm{A}$ 星的数据 产品包括一级数据产品, 大气产品和辐射产品等。本 文需要用到的风云四号产品包括成像仪全圆盘 $4 \mathrm{KML} 1$ 数据（以下简称： L1 数据）, 云检测实时产 品（以下简称：云检测数据）, 云相态实时产品（以 下简称：云相态数据）。

\section{2 数据集建立}

1.2.1 亮温云相态指数

风云四号属于静止轨道气象卫星, 具有对同一片
区域持续观测的优势。15 min 成像间隔可以忽略太阳 高度角对亮温和反射率的影响, 比较此时刻卫星云图 和 $15 \mathrm{~min}$ 前的卫星云图, 可以得到每个云像素点前后 $15 \mathrm{~min}$ 反射率和亮温的变化量。反射率只有光照时有 效, 亮温不受昼夜变化的影响, 使用亮温进行云相态 分类可以在昼夜间平滑过度。结合风云四号 $\mathrm{A}$ 星的通 道特点, 本文提出亮温云相态指数（Brightness temperature Cloud Phase Index，BTCPI），亮温云相态 指数用公式(1)表示:

$$
\mathrm{BT}_{\mathrm{CPI}}=\mathrm{BT}_{\text {now }}-\mathrm{BT}_{\text {past }}
$$

式中: $\mathrm{BT}_{\text {now }}$ 为当前图像云像素点对应的亮温值; $\mathrm{BT}_{\text {past }}$ 为 $15 \mathrm{~min}$ 前图像云像素点对应的亮温值。当云层移动 时, 遥感图像中的云区域从水云变成冰云, 或者从冰 云变成水云。因为冰云和水云在不同波段下的亮温值 不同 ${ }^{[10,13]}$, 相同波段下前后 $15 \mathrm{~min}$ 两张遥感图像的亮 温值相减结果与 0 比较即可检测出云相态。该公式只 针对 $15 \mathrm{~min}$ 前后都有云且云相态发生变化区域。若某 区域 $15 \mathrm{~min}$ 前后从无云变成有云、有云变成无云或云 相态不变, 亮温云相态指数则无法检测出该区域的云 相态。

\section{表 1 AGRI 通道成像设置}

Table 1 Imaging settings of AGRI channel

\begin{tabular}{ccc}
\hline Channel number & Central wavelength/ $\mu \mathrm{m}$ & Resolving power $/ \mathrm{km}$ \\
\hline 1 & 0.47 & 1.0 \\
2 & 0.65 & 0.5 \\
3 & 0.825 & 1.0 \\
4 & 1.375 & 2.0 \\
5 & 1.61 & 2.0 \\
6 & 2.25 & 2.0 \\
7 & $3.75 \mathrm{H}$ & 2.0 \\
8 & $3.75 \mathrm{~L}$ & 4.0 \\
9 & 6.25 & 4.0 \\
10 & 7.1 & 4.0 \\
11 & 8.5 & 4.0 \\
12 & 10.7 & 4.0 \\
13 & 12.0 & 4.0 \\
14 & 13.5 & 4.0 \\
\hline
\end{tabular}

为了减少噪声对亮温云相态指数的影响, 人为设 置一个常数 $\delta(\delta>0)$, 使分类结果更加准确, 公式(2) 如下:

$$
\text { Result }= \begin{cases}\mathrm{BT}_{\mathrm{CPI}}+\delta, & \mathrm{BT}_{\mathrm{CPI}}<0 \\ \mathrm{BT}_{\mathrm{CPI}}-\delta, & \mathrm{BT}_{\mathrm{CPI}}>0\end{cases}
$$

当冰云的亮温值低于水云时: $15 \mathrm{~min}$ 前图像 Result $<0$ 的云区域为的冰云, Result $>0$ 的云区域为水云。

风云四号 $\mathrm{A}$ 星亮温云相态指数的示意图如图 1 所 示。 


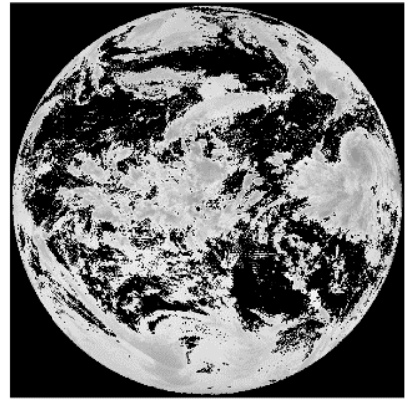

(a) 15 min 前云亮温图

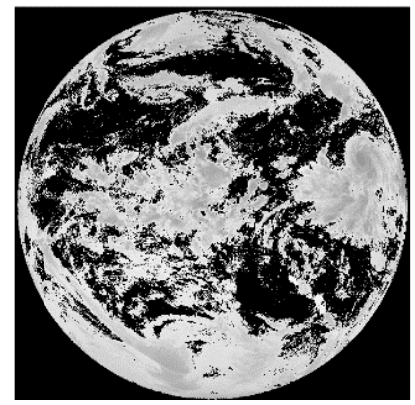

(b) 15 min 后云亮温图

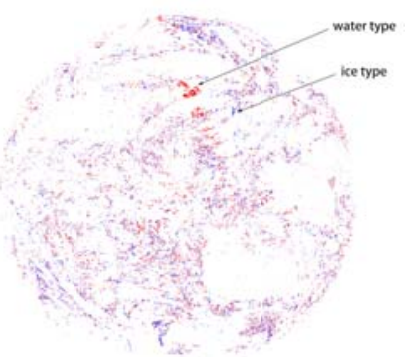

(c) 亮温云相态指数结果图

$\begin{array}{llll}\text { (a) Cloud map of bright temperature15 minutes ago } & \text { (b) Cloud map of bright temperature15 minutes later } & \text { (c) Result of BTCPI }\end{array}$

图 1 亮温云相态指数示意图

图 1 中(a)和(b)分别为风云四号卫星 $15 \mathrm{~min}$ 之前和 $15 \mathrm{~min}$ 后去除非云像素区域的亮温图, 黑色区域代表 无云。(c)为亮温云相态指数得到的 $15 \mathrm{~min}$ 前云相态数 据, 为了方便观察, 上色处理后, 红色代表水云, 蓝 色代表冰云。可以看出, 利用亮温云相态指数能够清 楚分类出部分冰云和水云。

\subsection{2 建立训练和预测数据集}

选取 L1 数据过程中, 考虑昼夜和四季的变换对 遥感影像遥感卫星云图的影响。本文选取不同季节、 昼夜下的数据, 以丰富数据集共计 48 个时刻。

L1 数据并不能直接用于云相态分类, 需要对该数 据进行预处理。预处理有 2 个目的：第一个利用云检 测数据去除遥感图像中无云区域的数据; 第二个是对 遥感图像有云的数据进行辐射定标。云检测是云相态 分类的基础和前提, 一般先进行云检测, 标记出卫星 云图中有云和无云的部分, 然后再进行云相态分类。 风云四号云检测方法可以参考高军等人的研究 ${ }^{[23]}$, 风 云四号官方也给出了云检测的结果, 本文直接使用官 方云检测数据。云检测完成后需要根据给定的辐射定 标表进行辐射定标。根据官方辐射定标表，表 1 中， FY-4A 卫星 1 到 6 号通道定标为反射率, 7 到 14 号通 道定标为亮温。

利用亮温云相态指数, 对 L1 数据进行处理, 构 建云相态数据集。为了弥补数据集较少的情况, 消除 训练数据集和预测数据集选取的偶然性, 利用 6 折交 叉验证 ${ }^{[24]}$ 的方法, 将构建好的云相态数据集分为 6 组, 5 组为训练集, 1 组为预测集。

\section{2 集成学习}

随机森林（Random Forest，RF）是集成学习代表 算法之一。随机森林由多棵决策树 ${ }^{[25]}$ 构成, 决策树是 一种重要的分类方法, 单一的决策树可能会发生过拟 合。随机森林中的决策树引入了随机属性选择, 传统 的决策树在当前特征中选取最优的特征开始进行划
Fig.1 Sketch map of BTCPI

分，在随机森林中决策树的每个结点都是从特征集合 中随机选择 $K$ 个特征子集, 然后从特征子集中选择最

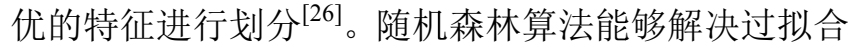
等问题, 还可以获得优越的泛化性能。集成学习示意 图如图 2 所示。

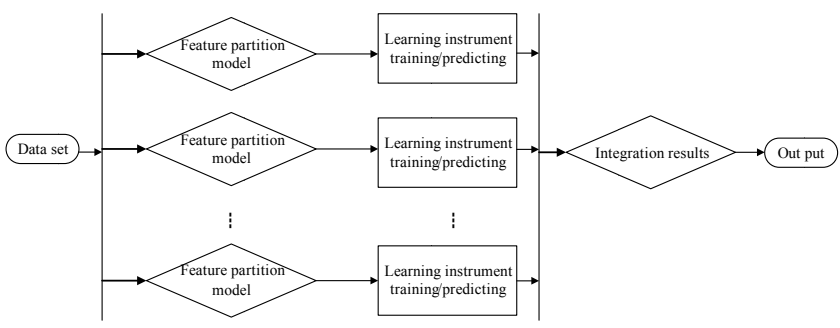

图 2 集成学习示意图

Fig.2 Sketch map of ensemble learning

\section{1 特征选取}

特征选取对训练机器学习算法有重要作用, 特征 选取不同可能影响最终建立模型与预测结果的好坏。 水和冰的折射率虚部 ${ }^{[27]}$ 能够表示水、冰粒子吸收辐射 的能力, 也是云相态分类的一种重要参考因子 ${ }^{[14-15]}$, 如图 3 所示。

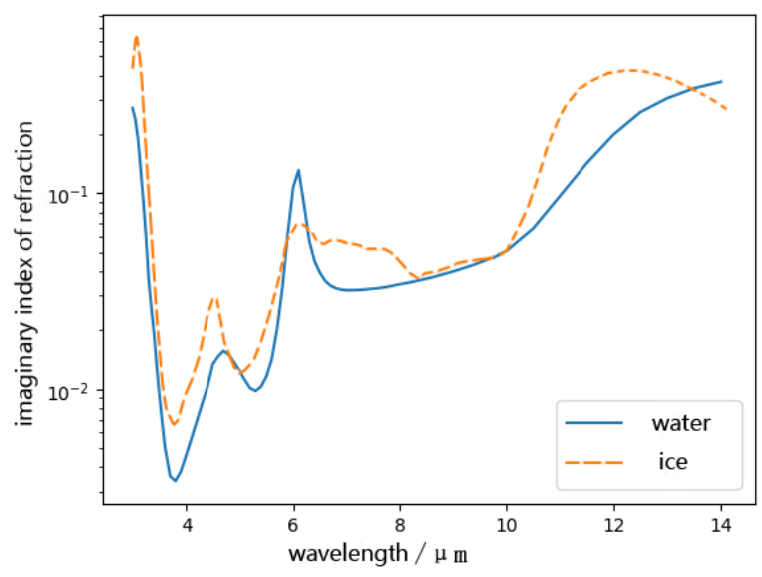

图 3 冰和水的折射率虚部随波长变化图

Fig.3 Imaginary index of refraction of water and ice across infrared wavelength

风云四号成像仪中有波长为 $6.25 \mu \mathrm{m} 、 7.1 \mu \mathrm{m}$ 和 
$12 \mu \mathrm{m} 、 13.5 \mu \mathrm{m}$ 通道, 从图 3 可以看出, $6.25 \mu \mathrm{m}$ 和 $7.1 \mu \mathrm{m}$ 连线斜率与 $12 \mu \mathrm{m}$ 和 $13.5 \mu \mathrm{m}$ 连线斜率在水态 和冰态下有差异, 因此可以根据斜率来区分冰云和水 云。定义水汽指数 $S_{7.1}$ 和热红外指数 $S_{13.5}$ 来描述折射 虚部的斜率不同，如公式(4)和公式(5)所示:

$$
\begin{gathered}
S_{7.1}=\frac{\mathrm{BT}_{7.1}-\mathrm{BT}_{6.25}}{7.1-6.25} \\
S_{13.5}=\frac{\mathrm{BT}_{13.5}-\mathrm{BT}_{12}}{13.5-12}
\end{gathered}
$$

结合其他云相态分类算法所使用的波段, 本文将 $8.5 \mu \mathrm{m}$ 和 $10.7 \mu \mathrm{m}$ 的亮温差 $\mathrm{BT}_{8.5}{ }_{10.7}, 10.7 \mu \mathrm{m}$ 和 $12 \mu \mathrm{m}$ 亮温差 $\mathrm{BT}_{10.7-12}$ 也作为分类的特征。集成学习特征选 取如下表 2 所示。

\section{表 2 云相态分类特征提取}

\begin{tabular}{|c|c|c|}
\hline Number & Feature name & Explain \\
\hline 1 & $\mathrm{BT}_{3.75 \mathrm{~L}}$ & $\begin{array}{l}\text { Brightness temperature of } \\
\text { central wavelength } 3.75 \mu \mathrm{m}\end{array}$ \\
\hline 2 & $\mathrm{BT}_{6.25}$ & $\begin{array}{l}\text { Bright temperature of central } \\
\text { wavelength } 6.25 \mu \mathrm{m}\end{array}$ \\
\hline 3 & $\mathrm{BT}_{7.1}$ & $\begin{array}{l}\text { Bright temperature of central } \\
\text { wavelength } 7.1 \mu \mathrm{m}\end{array}$ \\
\hline 4 & $\mathrm{BT}_{8.5}$ & $\begin{array}{l}\text { Bright temperature of central } \\
\text { wavelength } 8.5 \mu \mathrm{m}\end{array}$ \\
\hline 5 & $\mathrm{BT}_{10.7}$ & $\begin{array}{l}\text { Bright temperature of central } \\
\text { wavelength } 10.7 \mu \mathrm{m}\end{array}$ \\
\hline 6 & $\mathrm{BT}_{12.0}$ & $\begin{array}{l}\text { Bright temperature of central } \\
\text { wavelength } 12.0 \mu \mathrm{m}\end{array}$ \\
\hline 7 & $\mathrm{BT}_{13.5}$ & $\begin{array}{l}\text { Bright temperature of central } \\
\text { wavelength } 13.5 \mu \mathrm{m}\end{array}$ \\
\hline 8 & $\mathrm{BT}_{8.5 \_10.7}$ & $\begin{array}{l}\text { Bright temperature of central } \\
\text { wavelength } 6.25 \mu \mathrm{m} \text { and } 10.7 \\
\mu \mathrm{m}\end{array}$ \\
\hline 9 & $\mathrm{BT}_{10.7 \_12}$ & $\begin{array}{l}\text { Bright temperature of central } \\
\text { wavelength } 10.7 \mu \mathrm{m} \text { and } 12 \mu \mathrm{m}\end{array}$ \\
\hline 10 & $\mathrm{~S}_{7.1}($ Steam index $)$ & $\begin{array}{l}\text { Bright temperature change } \\
\text { rates of central wavelength } 7.1 \\
\mu \mathrm{m} \text { and } 6.25 \mu \mathrm{m}\end{array}$ \\
\hline 11 & $\begin{array}{l}\mathrm{S}_{13.5}(\text { Thermal } \\
\text { infrared index })\end{array}$ & $\begin{array}{l}\text { Bright temperature change } \\
\text { rates of central wavelength } \\
13.5 \mu \mathrm{m} \text { and } 12 \mu \mathrm{m}\end{array}$ \\
\hline
\end{tabular}

Table 2 Cloud phase classification feature extraction

\section{2 实验过程}

实验使用 scikit-learn 机器学习库进行随机森林的 平台搭建, 使用 10 棵决策树进行集成学习。随机森林 通过训练集形成云相态分类模型, 再利用分类模型对
预测集进行预测, 得到云像素点为水云和冰云的概率, 选取概率较大者为预测的结果。

\section{3 结果与讨论}

本文对云相态分类结果从两方面进行比较。一方 面从视觉上进行比较, 另一方面从数据上进行比较。

\section{1 视觉对比}

视觉对比法是直接通过人眼观察结果图像, 该方 法可以很直观地看出云相态分类的结果和每种云相态 的轮廓。

选取 2018 年 10 月 23 日 7 时（北京时间 2018 年 10 月 23 日 15 时) 的 FY-4A 遥感图像作为代表, 使 用本文算法对该遥感图像进行云相态分类, 得到的云 相态分类结果图和风云四号官方云相态产品图像、 FY-4A 通道合成图、Himawari-8 云产品图像进行比较。

图 4 为 3 种云相态分类算法的结果图和官方云相 态图。其中 (a)为 FY-4A 官方云相态图, (b)为本文算 法得到的云相态分类结果图, (c)为 BP 神经网络分类 结果图, (d)为阈值法分类结果图。由图 4(b) 可以看出, 本文算法能够将大部分的冰云和水云正确分类。 FY-4A 官方云相态产品把云分为液态水云、过冷水云、 混合云、冰云。本文将液态水云和过冷水云都归为水 云。官方云相态产品中有混合云, 所以混合云部分在 云相态分类结果(b)中被冰云或水云代替。

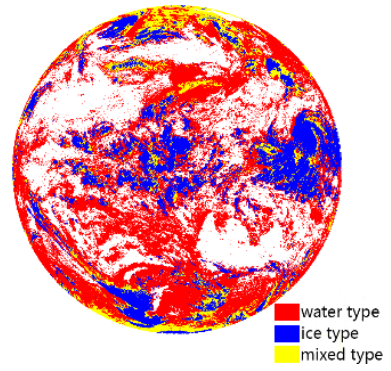

(a) 官方云相态产品

(a) Official cloud phase

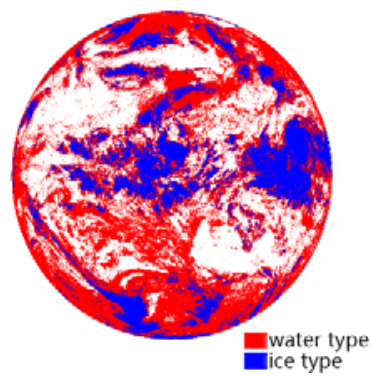

(c) BP 神经网络

(c) BP neural network

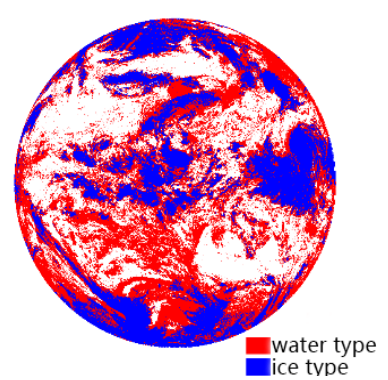

(b) 本文算法结果

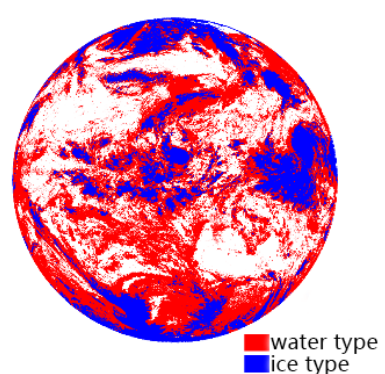

(d) 阈值法 (b) Method result map

图 4 不同算法云相态分类结果图

Fig.4 Cloud phase classification results of different algorithms 图 5 为本文算法结果与 FY-4A 通道合成图。 
FY-4A 通道合成图是利用通道 2, 通道 3 和通道 5 进 行图像合成。3 个通道的差异能够反映水体, 云和地 物信息, 由于水云在这 3 个通道的反射率接近, 在合 图像中为白色, 而积雪和冰云的信息在通道 5 的反射 率远远高于水, 图像中为蓝色调 ${ }^{[19]}$, 为了提高人眼的 观测性, 提高了亮度, 通道合成图像如图 5(a)所示。 利用本文算法得到的云相态分类结果如图 5(b)所示, 水云用红色标出, 冰云用蓝色标出。从整体看, 本文 算法的云相态分类结果在不同下垫面检测区域表现良 好。

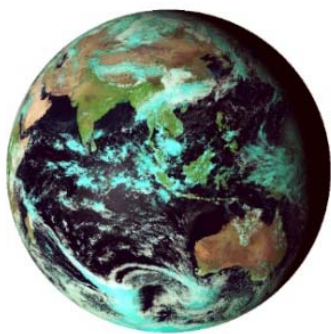

(a) 通道合成图

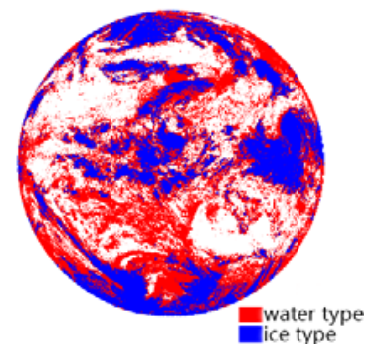

(b) 本文算法结果 (a) Channel synthesis map
混合云; 深对流云或高云、卷云为高云, 归类为冰云。

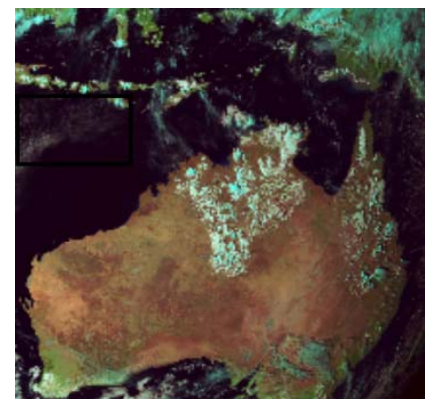

(a) 通道合成图

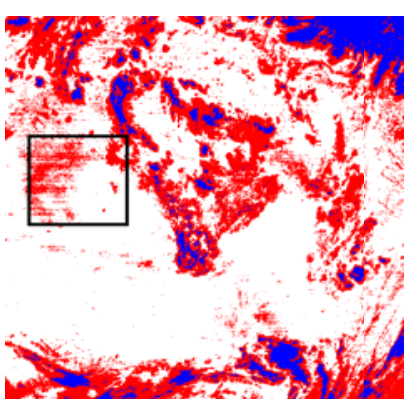

(b)本文算法结果

(b) Method result map (a) Channel synthesis map

图 7 澳大利亚附近区域的云相态情况

Fig.7 Cloud phase of nearby Australia area

中国南部及周边区域 Himawari-8 卫星的云分类 产品与云相态分类结果如图 8 所示。从图中可以看出, 由于多了混合云的存在，一部分冰云和水云被归为了 混合云, 但云相态分类的大致轮廓相似, 存在的水云 基本都被检测出来。

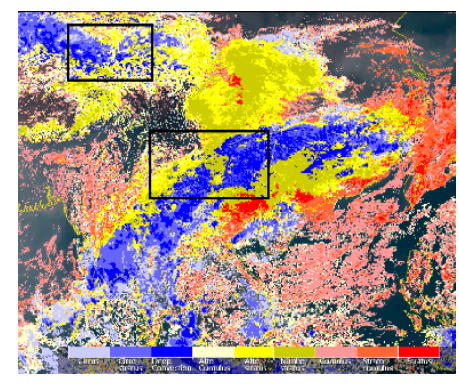

(a) Himawari-8 云分类图

(a) Himawari-8 result Map

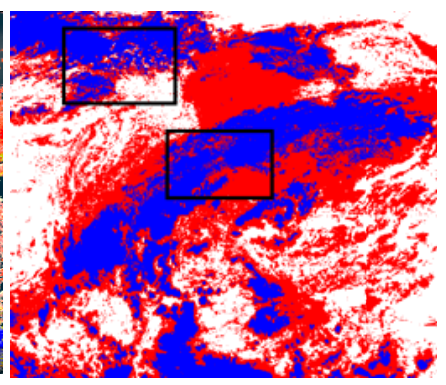

(b)本文算法结果
图 8 中国南部及周边区域的云相态情况

Fig.8 Cloud phase of southern china and its surrounding areas

\section{2 数据评估}

本文使用准确率、错误率、灵敏性和特效性 4 个

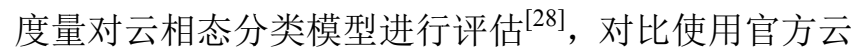
相态数据作为真实云相态数据。设水云为正元组, 冰 云为负元组, 准确率、错误率、灵敏性和特效性计算 公式如下:

$$
\begin{aligned}
& \text { accuracy }=\frac{T P+T N}{P+N} \\
& \text { error rate }=\frac{F P+F N}{P+N} \\
& \text { sensitivity }=\frac{T P}{P} \\
& \text { specificity }=\frac{T N}{N}
\end{aligned}
$$

式中: TP、TN、FP、FN、P、N 分别表示真正例、真 负例、假正例、假负例、正样本数和负样本数。本文 水云; 雨层云、高层云、高积云为中云, 归类为冰水 
算法, BP 神经网络, 阈值法云相态分类模型 6 次交叉 验证评估均值详情如表 3 所示。

\section{表 3 云相态分类模型评估详情}

Table 3 Details of cloud phase state classification model assessment

\begin{tabular}{lcccc}
\hline Algorithm & Accuracy & Error Rate & Sensitivity & Specificity \\
\hline $\begin{array}{l}\text { ensemble } \\
\text { learning }\end{array}$ & $86.63 \%$ & $13.37 \%$ & $91.69 \%$ & $76.10 \%$ \\
$\begin{array}{l}\text { BP neural } \\
\text { network }\end{array}$ & $79.93 \%$ & $20.07 \%$ & $79.10 \%$ & $82.23 \%$ \\
$\begin{array}{l}\text { threshold } \\
\text { method }\end{array}$ & $76.38 \%$ & $23.62 \%$ & $83.48 \%$ & $55.75 \%$ \\
\hline
\end{tabular}

从表 3 评估结果可以看出, 本文算法结果准确率 超过了其他两种分类算法, 分类模型效果良好。

\section{4 结论}

本文提出的基于集成学习的云相态分类算法, 针 对 FY-4 遥感图像, 该算法可以提供相对良好的云相 态分类结果。该算法的关键是利用亮温云相态指数构 建云相态数据集。相对于传统的人工标注法和阈值法 为数据贴标签, 亮温云相态指数对 $\mathrm{L} 1$ 数据进行初步 云相态分类进而产生大量标签且该方法对使用者先验 知识要求不高。分类特征的提取主要利用不同波段之 间对应冰云和水云的亮温值差异。随机森林利用训练 集训练最后建立云相态分类模型, 经过评估, 该模型 的分类准确率较好, 可以进行快速云相态分类。云相 态结果图在视觉上和官方结果图大致相当, 能够反映 不同区域、水云冰云交界处的云相态特征, 对于一些 细小的薄云也能够正确分类, 总体分类效果良好, 能 为后续其他云数据反演工作提供参考依据。同时, 本 文算法对云相态只划分为水云和冰云, 下一步工作中, 将针对混合云相态的划分进行研究。

\section{参考文献:}

[1] Shupe M D. Clouds at Arctic Atmospheric Observatories. Part II: Thermodynamic Phase Characteristics[J]. Journal of Applied Meteorology \& Climatology, 2011, 50(3): 645-661.

[2] Knap W H, Stammes P, Koelemeijer R B A. Cloud Thermodynamic-Phase Determination From Near-Infrared Spectra of Reflected Sunlight[J]. Journal of the Atmospheric Sciences, 2002, 59(59): 83-96.

[3] 任建奇, 严卫, 叶晶, 等. 云相态的卫星遥感研究进展 [J]. 地球科学 进展, 2010, 25(10): 1051-1060.

REN Jianqi, YAN Wei, YE Jing. Advances in Satellite Remote Sensing of Cloud Phase State[J]. Advances in Earth Science, 2010, 25(10):
1051-1060

[4] Ackerman S A, Smith W L, Revercomb H E, et al. The 27-28 October 1986 FIRE IFO Cirrus Case Study: Spectral Properties of Cirrus Clouds in the 8-12 $\mu \mathrm{m}$ Window[J]. Monthly Weather Review, 2009, 118(118): 2377-2388.

[5] Rossow W B, Schiffer R A. Advances in understanding clouds from ISCCP[J]. Bull.amer.meteor.soc, 1999, 80(11): 2261-2287.

[6] King M D, Platnick S, Yang P. Remote Sensing of Liquid Water and Ice Cloud Optical Thickness and Effective Radius in the Arctic: Application of Airborne Multispectral MAS Data[J]. Journal of Atmospheric \& Oceanic Technology, 2004, 21(6): 857-875.

[7] WANG J, CHAO L, MIN M. Effects and Applications of Satellite Radiometer 2.25- $\mu \mathrm{m}$ Channel on Cloud Property Retrievals[J]. IEEE Transactions on Geoscience \& Remote Sensing, 2018, 99: 1-10.

[8] Arking A, Childs J D. Retrieval of Cloud Cover Parameters from Multispectral Satellite Images[J]. Journal of Applied Meteorology, 2003, 24(4): 322-333.

[9] 刘健, 董超华, 朱元竞, 等. FY-1C 资料在云顶粒子热力学相态分析 中的应用研究[J]. 大气科学, 2003, 27(5): 901-908.

LIU Jian, DONG Chaohua, ZHU Yuanjing. Thermodynamic Phase Analysis of Cloud Particles with FY-1C Data[J]. Chinese Journal of Atmospheric Sciences, 2003, 27(5): 901-908.

[10] 刘健, 李云. 风云二号静止气象卫星的云相态识别算法[J]. 红外与毫 米波学报, 2011, 30(4): 322-327.

LIU Jian, LI Yun. Cloud phase detection algorithm for geostationary satellite data[J]. Journal of Infrared and Millimeter Waves, 2011, 30(4): 322-327.

[11] MOURI K, IZUMI T, SUZUE H. Algorithm Theoretical Basis Document for Cloud Type/Phase Product[EB/OL]. http://www.data. jma.go.jp/mscweb/technotes/msctechrep61-2.pdf. 2016.

[12] 郭洪涛, 谢欢欢, 马英, 等. 基于支持向量机的云相态分析 [J]. 解放 军理工大学学报: 自然科学版, 2012, 13(2): 226-231.

GUO Hongtao, XIE Huanhuan, MA Ying. Analysis of Cloud Phase Based on Support Vector Machine[J]. Journal of PLA University of Science and Technology: Natural Science Edition, 2012, 13(2): 226-231.

[13] 熊贤成, 杨春平, 敖明武, 等. 基于 BP 神经网络的云相态检测方法研 究[J]. 遥感技术与应用, 2015,30(4): 714-718.

XIONG Xiancheng, YANG Chunping, AO Mingwu. AResearch on Cloud Phase Detection Based on BPNetural Network[J]. Remote Sensing Technology and Application, 2015, 30(4): 714-718.

[14] 李锡祥, 麻金继, 梁晓芳. 基于 BP 神经网络进行云相态识别方法的 研究 $[J]$. 大气与环境光学学报, 2010, 5(4): 299-304.

LI Xixiang, MA Jinji, LIANG Xiaofang. Retrieving Cloud Phase Based on BP Neural Network[J]. Journal of Atmospheric and Environmental Optics, 2010, 5(4): 299-304 
[15] 靳泽群, 张玲, 刘神聪. 基于 BP 神经网络的云检测和云相态识别 [J]. 光学与光电技术, 2016, 14(5): 74-77.

JIN Zequn, ZHANG Ling, LIU Shencong. Cloud Detection and Cloud Phase Retrieval Based on BPNeural Network[J]. Optics\& Optoelectronic Technology, 2016, 14(5): 74-77.

[16] 盛夏, 孙龙祥, 郑庆梅. 模拟退火优化 BP 神经网络进行云相态分类 [J]. 解放军理工大学学报: 自然科学版, 2008, 9(1): 98-102. SHENG Xia, SUN Longxiang, ZHENG Qingmei. Simulated Annealing Optimized BP-ANN Method for Cloud Thermodynamic Phase Retrieval[J]. Journal of PLA University of Science and Technology: Natural Science Edition, 2008, 9(1): 98-102.

[17] 郭晶, 杨春平, 叶玉堂, 等. SOFM 神经网络的 FY-3A/VIRR 多光谱图 像云相态反演方法 [J]. 光电工程, 2015, 42(12): 20-24.

GUO Jing, YANG Chunping, YE Yutang. A Cloud Phase Retrieval Approach Based on SOFM Neural Network Using FY-3A/VIRR Multi-channel Images[J]. Opto-Electronic Engineering, 2015, 42(12): 20-24.

[18] 张鹏, 郭强, 陈博洋, 等. 我国风云四号气象卫星与日本 Himawari-8/9 卫星比较分析 [J]. 气象科技进展, 2016, 6(1): 72-75.

ZHANG Peng, GUO Qiang, CHEN Boyang. The Chinese Next-Generation GeostationaryMeteorological Satellite FY-4 Compared with theJapanese Himawari-8/9 Satellites[J]. Advances in Meteorological Science and Technology, 2016, 6(1): 72-75.

[19] 陆风, 张晓虎, 陈博洋, 等. 风云四号气象卫星成像特性及其应用前 景[J]. 海洋气象学报, 2017, 37(2): 1-12.

LU Feng, ZHANG Xiaohu, CHEN Boyang. FY-4 geostationary meteorological satellite imaging characteristics and its application prospects[J]. Journal of Marine Meteorology, 2017, 37(2): 1-12.

[20］董瑶海. 风云四号气象卫星及其应用展望[J]. 上海航天, 2016, 33(2): $1-8$.

DONG Yaohai. FY-4 Meteorological Satellite and its Application
Prospect[J]. Aerospace Shanghai, 2016, 33(2): 1-8.

[21] 华建文, 毛建华. “风云四号”气象卫星大气垂直探测仪[J]. 科学, 2018, 70(1): 24-29.

HUA Jianwen, Mao Jianhua. "FY-4" MeteorologicalSatellite Atmospheric Vertical Detector[J]. Science, 2018, 70(1): 24-29.

[22] 王洤泉, 沈霞. 风云四号辐射成像仪及其数据在卫星气象中的应用 [J]. 自然杂志, 2018, 40(1): 1-11.

WANG Ganquan, SHEN Xia. The FY-4 Radiometer Imager and The Application of its Data in the Satellite Meteorology[J]. Chinese Journal of Nature, 2018, 40(1): 1-11

[23] 高军, 王恺, 田晓宇, 等. 基于 BP 神经网络的风云四号遥感图像云检 测算法[J]. 红外与毫米波学报, 2018, 37(4): 477-485.

GAO Jun, WANG Kai, TIAN Xiaoyu. A BP-NN based cloud detection method for FY-4 remote sensing images[J]. Journal of Infrared and Millimeter Waves, 2018, 37(4): 477-485.

[24] 范永东. 模型选择中的交叉验证方法综述[D]. 太原: 山西大学, 2013. FAN Yongdong. Overview of cross-validation methods in model selection[D]. Taiyuan: Shanxi University, 2013.

[25] 亱丽华, 吉根林. 决策树分类技术研究 [J]. 计算机工程, 2004, 30(9): 94-96.

LUAN Lihua, JI Genlin. The Study on Decision Tree Classification Techniques[J]. Computer Engineering, 2004, 30(9): 94-96.

[26] 周志华. 机器学习[M]. 北京: 清华大学出版社, 2016. ZHOU Zhihua. Machine Learning[M]. Beijing: Tsinghua Press, 2016.

[27] Strabala K I, Ackerman S A, Menzel W P. Cloud properties inferred from 8-12î1/4m data[J]. Journal of Applied Meteorology, 1994, 33(2): $212-229$.

[28] HAN Jiawei, Micheline Kamber, PEI Jian. 数据挖掘概念与技术[M]. 3 版, 北京: 机械工业出版社 2012 .

HAN Jiawei, Micheline Kamber, PEI Jian. Data Mining[M]. Third Edition, Beijing: China machine press, 2012. 